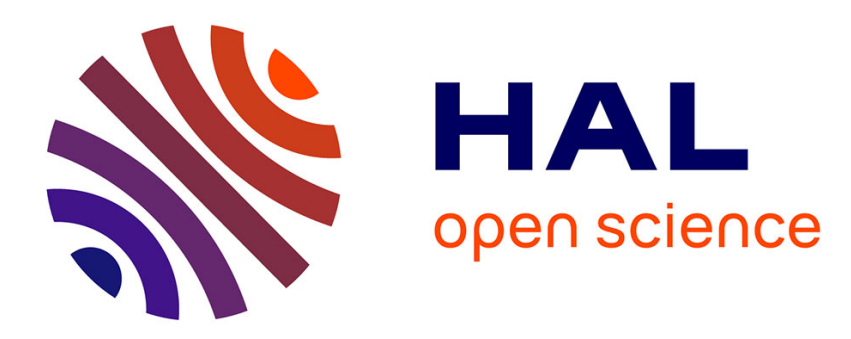

\title{
Exosomes as intercellular signalosomes and pharmacological effectors
}

\author{
Michel Record
}

\section{To cite this version:}

Michel Record. Exosomes as intercellular signalosomes and pharmacological effectors. Biochemical Pharmacology, 2011, 81 (10), pp.1171. 10.1016/j.bcp.2011.02.011 . hal-00690186

\section{HAL Id: hal-00690186 https://hal.science/hal-00690186}

Submitted on 22 Apr 2012

HAL is a multi-disciplinary open access archive for the deposit and dissemination of scientific research documents, whether they are published or not. The documents may come from teaching and research institutions in France or abroad, or from public or private research centers.
L'archive ouverte pluridisciplinaire HAL, est destinée au dépôt et à la diffusion de documents scientifiques de niveau recherche, publiés ou non, émanant des établissements d'enseignement et de recherche français ou étrangers, des laboratoires publics ou privés. 


\section{Accepted Manuscript}

Title: Exosomes as intercellular signalosomes and pharmacological effectors

Author: Michel Record

PII: $\quad$ S0006-2952(11)00115-8

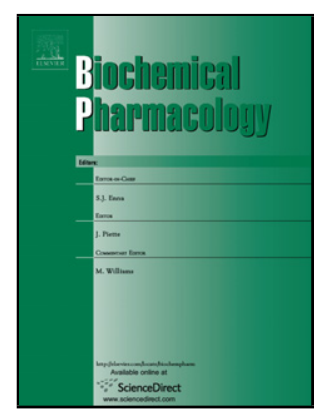

DOI: doi:10.1016/j.bcp.2011.02.011

Reference: BCP 10832

To appear in:

$B C P$

Received date:

13-12-2010

Revised date:

14-2-2011

Accepted date:

$17-2-2011$

Please cite this article as: Record M, Exosomes as intercellular signalosomes and pharmacological effectors, Biochemical Pharmacology (2010), doi:10.1016/j.bcp.2011.02.011

This is a PDF file of an unedited manuscript that has been accepted for publication. As a service to our customers we are providing this early version of the manuscript. The manuscript will undergo copyediting, typesetting, and review of the resulting proof before it is published in its final form. Please note that during the production process errors may be discovered which could affect the content, and all legal disclaimers that apply to the journal pertain. 


\title{
Exosomes as intercellular signalosomes and pharmacological effectors
}

\author{
Michel Record $(*)$, Caroline Subra, Sandrine Silvente-Poirot, Marc Poirot
}

\begin{abstract}
INSERM-UMR 1037, Cancer Research Center of Toulouse (CRCT), Team« Sterol Metabolism and Therapeutic Innovation in Oncology " Institut Claudius Regaud, 20-24 Rue du Pont Saint-Pierre, 31052 Toulouse Cedex, France ; and Université Paul Sabatier, 118 Route de Narbonne, Toulouse, France
\end{abstract}

\section{Running title : Exosomes as vectorized signaling}

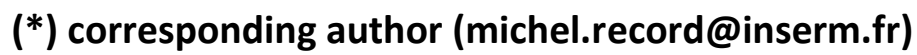

\begin{abstract}
.
Cell secretion is a general process involved in various biological responses. Exosomes are part of this process and have gained considerable scientific interest in the past five years. Several steps through investigations across the last 20 years can explain this interest. First characterized during reticulocyte maturation, they were next characterized as a key player in the immune response and cancer immunotherapy. More recently they were characterized as vectors of mRNAs, miRNAs and also lipid mediators able to act on target cells. They are the only type of vesicles released from an intracellular compartment from cells in viable conditions. They appear as a vectorized signaling system operating from inside a donor cell towards either the periphery, the cytosol, or possibly to the nucleus of target cells. Exosomes from normal cells trigger positive effects, whereas those from pathological ones, such as tumor cells or infected ones may trigger non-positive health effects. Therefore regulating the biogenesis and secretion of exosomes appear as a pharmacological challenge to intervene in various pathophysiologies. Exosome biogenesis and molecular content, interaction with target cells, utilisation as biomarkers, and functional effects in various pathophysiologies are considered in this review.
\end{abstract}

\section{Introduction: the exosome pathway}

Exosomes are nanovesicles $(50-100 \mathrm{~nm})$ released from viable cells, either constitutively or upon activation of cell secretion, but neither from lysed nor apoptotic cells. They are not released from plasma membrane shedding but they are secreted from an intracellular compartment, the MultiVesicular Bodies (MVB), or late endosomes $[1,2]$. 


\section{A. Biogenesis and release of exosomes by producer cells: from inside the cell to an extracellular network (Figure 1)}

Exosomes bear specific protein markers such as tetraspanins (CD63,CD9,CD81), HeatShock proteins (HSP 70) or the Transferin Receptor in the case of reticulocyte (TfR), that make the exosomes distincts from vesicles released upon plasma membrane shedding and called ectosomes [3]. These latter contain distinct markers such as glycophorin. Exosomes are also totally distincts from apoptotic microparticles which bear markers like CD31 or Annexin $\mathrm{V}$ [4].

They have been described for the first time by Jonhstone and collaborators in 1987 during the reticulocytes maturation process [1]. Initially, exosomes were thought to be implicated in removing unneeded membrane proteins from cells that have poor capacities to degrade proteins with a lysosomal system, or cells which are located towards a drainage system such as the tubule kidney or gut [5]. Since then, several cell types have been described to release exosomes in extracellular medium in-vitro as hematopoietic cells (B cells, T cells, Dendritic cells, mast cells, platelets), intestinal epithelial cells, Schwann cells, neuronal cells, adipocytes, fibroblast (NIH3T3), tumor cells... Exosomes are found in- vivo in several biological fluids such as urine, plasma, epididymal fluid, amniotic liquid, malignant and pleural effusions of ascites, bronchoalveolar lavage fluid, synovial fluid and breast milk.

They feature a density ranging from $1.13 \mathrm{~g} / \mathrm{ml}$ for $B$ cell exosomes [2] to $1.19 \mathrm{~g} / \mathrm{ml}$ for epithelial intestinal cell exosomes [6].

The initial step in exosome biogenesis is the intralumenal vesicle (ILV) formation by inward budding of the MVB membrane inside the cell [7]. The resulting vesicles contain cytosol and expose internal membrane of the MVB at their surface. Subsequently, the repartition of proteins between the two leaflets of the plasma membrane should be maintained in the ILVs, which progressively accumulate during endosome maturation. However phospholipid asymmetry may be not preserved during ILV formation since exosomes appear to have a similar lipid composition between the two membrane leaflet [8], consistent with the presence of a phospholipid scramblase [9].Thus, late endosomes can accumulate up to hundred intraluminal vesicles (ILVs) [10]. Similar conclusion can be drawn from observations performed in the mast cell line RBL-2H3 [9]. This type of cell contains about $30 \mathrm{MVBs}[9]^{(\mathrm{a})}$, which means that one RBL-2H3 cell could contain $3000 \mathrm{ILVs}$, providing that all MVBs were filled. Actually ILV release is sectorized in the cell, i.e is limited in some region of the cell periphery, indicating that the whole MVB cell content is not released at once.

(a) 14 intralumenal vesicles (ILVs) of about $70 \mathrm{~nm}$ of diameter were observed inside an electron microscopy focal plan of $70 \mathrm{~nm}$ thick (see [9] supplementary data Fig S1). Thus a $600 \mathrm{~nm}$ average diameter MVB [9], corresponding to about 8 focal plans, contains a 100 of ILVs, i.e exosomes. 


\section{Sorting proteins in exosomes:}

\subsection{Sorting via the Endosomal Sorting Complex Required for Transport (ESCRT)} machinery. The sorting of protein complexes into MVB vesicles is a highly regulated process and a variety of studies indicate that monoubiquitination serves as a signal that directs protein complexes into the MVB pathway. The Endosomal Sorting Complex required for Transport (ESCRT) play a role in the ILV formation. Some of the ESCRT proteins and ESCRTassociated proteins such as Tsg101 and Alix are recovered in exosomes.

The ESCRT machinery is transiently recruited to the cytosolic side of the endosomal membrane for sorting of selected protein to ILV and for ILV formation [11]. The ESCRT machinery is constituted of three separate protein complexes called ESCRT -I, ESCRT- II, ESCRT -III .

Vps27/Hrs recruits Tsg101 and its complex the ESCRT -I which binds the ubiquitinated cargo protein and activates ESCRT II. Tsg101 then recruits ESCRT - III via ESCRT -II or AIP1(Alix). Indeed, ESCRT- II initiates the oligomerization and the formation of the ESCRT- III complex that seems to have a function in the concentration of MVB proteins and sequesters proteins into the inward budding vesicles of the MVB. Then ESCRT- III recruits the deubiquitinating enzyme Doa4 which removes the ubiquitin tag from the cargo protein prior to sorting into the MVB vesicles. Finally, a multimeric AAA-type (ATPase associated with various cellular activities) ATPase Vps4 binds to ESCRT - III and disassembles the ESCRT III complex in an ATP-dependent manner $[12,13]$.

The ESCRT-associated protein AIP1/Alix has also been implicated in intraluminal vesicle formation. It has been demonstrated that Alix associates with a specific lipid of the late endosomes called Lyso-bisphosphatidic acid [(LBPA), or BMP for bis(monacylglycero)phosphate], in reconstituted liposomes with an interior low $\mathrm{pH}$ [14] . LBPA has been shown to be located mainly in late endosomes and ILV [15].

Some proteins without ubiquitination interact with component of ESCRT complex and are engulfed in exosomes. The transferin receptor (TfR) can associate with Alix for instance, suggesting that different protein interactions can provide access to the final steps of ESCRT processing [16].

\subsection{Sorting via an ESCRT independent sorting process.}

1.2.1. Sorting by protein incorporation into detergent-resistant protein complexes.

In immature dendritic cells (DC), an alternative MVB sorting mechanism for MHC class II in antigens loaded DCs was observed in response to cognate interactions with $\mathrm{T}$ cells [17]. This pathway is characterized by the incorporation of MHC class II in detergentresistant protein complexes. MHC class II is sorted in MVB to ILVs for lysosomal targeting in a process that is driven by ubiquitination of the cytoplasmic domain of MHC class II. When DC interacts with a cognate T cell, ubiquitination is blocked and $\mathrm{MHC}$ class II is sorted together with CD9 into ILVs of the MVB resulting in secretion via exosomes [17]. Two distinct MVB pathways were characterized: one for lysosomal targeting and the other for exosome secretion. 


\subsubsection{Sorting by lipid affinity.}

Tetraspan protein (tetraspanins) have a high affinity for cholesterol and sphingolipids such as ceramides which may create domains as it occurs in membrane models. Tetraspanins and others proteins may partition into these domains because of their intrinsic physical properties and therefore into the budding vesicles. $[18,19]$

It has been evidenced for instance, that in Oli-neu cells (mouse oligodendroglial cell line, myelinating cells of the central nervous system) the proteolipid protein PLP (a major component of myelin) is released in association with exosomes. PLP is segregated into distinct subdomains on the MVB membrane and their transfer in exosomes is independent of the ESCRT machinery but requires the sphingolipid ceramide. They provide an alternative pathway for sorting cargo into MVB, that seems to depend on raft-based microdomains [18, 19].

\subsubsection{Sorting by protein affinity.}

Tetraspanins are known to form oligomers with other tetraspanins or with other proteins (transmembrane or cytosolic) and to participate to protein segregation. Tetraspanins associate with proteins such as integrins, growth factor receptors, MHC class II and the costimulator CD86. Other type of protein interactions between heat-shock proteins Hsp70 and Hsc70 and the transferin receptor (TfR) allow proteins to be addressed in exosomes [20].

\subsubsection{Higher-order oligomerization.}

A different type of exosomal protein-sorting pathway in Jurkat $\mathrm{T}$ cells that selectively incorporates proteins into exosomes has been described. Protein targeting operates on the basis of higher-order oligomerization (i.e the aggregation of oligomers) without regard to protein function or sequence, or mechanism of membrane association. This sorting mechanism concerns proteins which tend to form aggregates in the cells, and appears independent of the vacuolar protein-sorting machinery (VPS)[21 ].

\subsubsection{Incorporation of cytosolic proteins into exosomes.}

This engulfment of cytosolic proteins results of a microautophagy process [22], and appears to involve proteins located close to the MVB outer membrane during its inward budding, which could explain the presence of mRNAs and miRNAs in exosomes [23]. In that respect two major components of the RNA-Induced Silencing Complex (RISC) have been shown to associate with MVB and to be sorted into exosomes [24].

\section{Sorting lipids in exosomes (Figure 2)}

Exosomes display a specific lipidic composition which is distinct from that of the parental MVB. As for proteins, lipid content of exosomes presents some common features whatever the cell type they are released from, but also reflects partly their parental cells. Thus lipid sorting also occurs during exosome biogenesis. Localization of lipids depends upon the respective intracellular localization of biosynthetic enzymes, transporters, and catabolic enzymes. The MVB specific lipid named lysobisphosphatidic (LBPA, or BMP) interacts 
strongly with Alix, a protein involved in exosome biogenesis. Knocking down Alix expression triggers the diminution of the number of MVB in cells. However LBPA by itself when added to a lipid mixture mimicking the composition of the MVB membrane can induce internal budding of small vesicles from large liposomes [14]. Exosomes appeared not to be enriched in LBPA as compared with parental cells $[8,25]$. By remaining on the MVB membrane, LBPA might reach the critical concentration allowing its fusogenic properties [26] with subsequent fission of the nascent intralumenal vesicle (ILV). Also LBPA appears to control the back-fusion process of ILVs with the endosome membrane, since the number of ILVs is maintained in a dynamic state between formation and disappearance [27]. LBPA is biosynthesized from phosphatidylglycerol (PG) whose biosynthesis occurs in the mitochondria. PG would then be transported to the MVB (Fig 2) which appears to contain the conversion machinery of PG into LBPA since adding PG-containing liposomes in the endocytic track enhances the amount of LBPA recovered in MVB [28].

It was shown that knocking down Alix induces a decrease of the LBPA (BMP) level and reduces the number of intraluminal vesicles in late endosomes, concomitant with a decrease of cellular cholesterol rate [29] without affecting LDL endocytosis and cholesterol synthesis. Authors explained the decrease of cellular cholesterol by an incapacity of MVB to store and retain cholesterol given the reduced amount of intralumenal vesicles, suggesting that LBPA controls the cholesterol capacity of endosomes. The exogenously addition of LBPA in Alix knocked down cells restores the internal vesicles and cellular cholesterol level. It has been shown that LBPA is limiting in Niemann-Pick type C (NPC) cells characterized by substantial cholesterol accumulation in these endosomes, since LBPA addition can reverse this cholesterol accumulation [29]. LBPA strongly enhances the function of the transporter NPC2 in cholesterol transfer potentiated by ceramide, and also promotes NPC2-mediated membrane fusion [30]. Treatment of normal cells with an anti-LBPA antibody however results in a NPC-like phenotype suggesting that the antibody might prevent LBPA-cholesterol interaction. The hydroxyl group of the ester bond linking the fatty acid at the glycerol backbone of the LBPA molecule appears critical for LBPA-induced cholesterol redistribution which emphasize a key role of ILV in that process [29].

Interestingly, bioactive lipids such as prostaglandins are sorted into exosomes[9]. Those lipidic mediators display multiple biological effects related to inflammation. They are not produced in the MVB, but in the endoplasmic reticulum from where two key enzymes for their biosynthesis, Cox 1 and Cox 2 , are sorted into exosomes [9]. Only few proteins from endoplasmic reticulum are recovered in exosomes. However the presence of another enzyme of the prostaglandin biosynthesis, the PGE synthase, has been reported [23]. Also exosomes were shown to carry the PPAR $\gamma$ nuclear receptor modulator 15d-PGJ2 [9]. PPAR $\gamma$ regulates cholesterol homeostasis, and cholesterol ester have been shown to trigger cell proliferation [31]. Cholesterol biosynthesis involves enzymes organized at the site of the Anti Estrogen Binding Site (AEBS). AEBS is a molecular complex constituted by two cholesterogenic enzymes, the DHCR7 reductase and the D8D7I isomerase, whose association makes a third catalytic activity, namely the cholesterol epoxyde hydrolase [32]. Ligands of AEBS trigger macroautophagy in tumor cells through modulation of cholesterol metabolism [33], and a link between microautophagy of the late endosome compartment and exosome 
production has been proposed [22], suggesting a role of cholesterol in exosome biogenesis. Noteworthy, the ATPase Vps4 which binds ECRTIII in the ESCRT machinery (see§ 1.1) also interacts with oxysterol binding proteins and sterol metabolism [34]. Therefore investigating cholesterol metabolism in relation with exosome production should bring new insights in that field.

In conclusion, lipid sorting as well as protein sorting leads to a specific content of exosomes as compared with parental cells and other cellular structures.

\section{Exosome Secretion}

Two types of secretion mechanisms have been characterized, i.e secretion of exosomes can be constitutive or inducible, depending on the cell type and on the activation state of the cell [35].

\subsection{Constitutive secretion (Figure 3)}

Proteins destinated to the cell surface or to be secreted into the extracellular medium can be routed from the Trans Golgi Network (TGN) by an ubiquitary constitutive pathway that does not require a specific stimulus, albeit controlled according to cell activity (intracellular signalling, cell growth, differentiation...). Structures involved in this anterograde pathway are pleiomorphic secretory vesicules and tubules ranging from $50 \mathrm{~nm}$ $200 \mathrm{~nm}$ up to $2 \mu \mathrm{m}$ in length and leave the TGN, move through the cytoplasm along cytoskeletal tracks and fuse with plasma membrane [36]. Some Rab GTPases have been identified in the regulation of the traffic from TGN to plasma membrane such as Rab8, rab13 and Rab11. In the same manner, heterotrimeric G-protein and protein kinase D (PDK1-2) are thought to be involved [36]. During constitutive secretion, exosomes are transported within vesicles containing only one or two exosomes. An illustration of this process is reported in Fig 3.

\subsection{Regulated secretion}

This secretion pathway is regulated by various cell activation processes. Changes in intracellular calcium in mast cells and in human erythroleukemia cell line [37] induced exosome release. Also cell depolarisation induced by $\mathrm{K}^{+}$appears to increase the secretion of neuronal exosomes.

Cell activation by cross-linking of receptors such as IgE in mast cells or CD3 in T cells triggers secretion with release of exosomes. This process involves the small GTPase Rab11 and the citron kinase, a rhoA effector [38,39], and recently rab27 [40]. Other Rab GTPases associated with the endocytic system, Rab 7 and Rab 11, did not modify exosome secretion, suggesting that a specific subset of multivesicular endosomes participates in the generation of exosomes. Also a brefeldin A-inhibited guanine-nucleotide exchange protein 2 (BIG2 or ARFGEF2) has been proposed to have a role in the secretion of exosomes or exosome-like vesicles [35].

Exosomes secretion from immature dendritic cells (imDCs) can be transiently increased by $T$ cell clones interaction or cognate $C D 4^{+} T$ cells $[17,35]$. Also Ag specific $C D 4^{+} T$ cells 
stimulates exosome release by activated B cells [41]. On the opposite, another type of stimulus such as LPS decrease the release of exosomes from activated DC [42].

Among receptor-mediated exosome release, the GPCR-type receptor related to adenosine triphosphate (ATP)-gated ion channels, namely P2X7 receptor, susceptible to rapid changes in ionic fluxes, regulate the release of exosomes in immune and inflammatory effector cells [43].

Exosomes production appears as a tightly controlled biological process dependent upon p53 regulated transcription genes such as the Tumor Supression Activated Pathway 6 (TSAP6) [44]. Thus, TSAP6 has been shown to increase the exosome secretion following stress events such as those inducing DNA damage (senescence, radiations ...) [44].

Lipids are also involved in the regulation of exosome secretion. The lipid mediator phosphatidic acid originating either from the activity of the diacylglycerol kinase [45] or from phospholipase D (PLD2) activities promotes exosome secretion [46].

\section{B. Exosomes exhibit a specific content (Figure 4).}

\section{Molecular composition of exosomes.}

\subsection{Protein content}

Exosomes protein composition analyses have revealed both an ubiquitary composition and a cell type-specific protein composition. Most of the exosomal proteins that have been identified are found in parent's cell membranes of endocytic compartment or the plasma membrane, also in the cytosol, the Golgi, even the nucleus, but rarely the endoplasmic reticulum or mitochondria. Exosomes contain both typical membrane proteins like GPIanchored proteins, CD63 and Tumor Necrosis Factor Receptor 1 (TNFR1), and typical luminal proteins as annexin 2 and cytokines. Types of molecules involved are related to antigenpresentation (MHC-I, MHC-II) an co-presentation molecules (CD86), cell adhesion (Integrins, MFGE8...), cell structure and motility (actins, myosin, tubulin...), Heat shock proteins and chaperones (HSP70, HSP90), metabolic enzymes ( $\beta$-Enolase, fatty acid synthase, Glyceraldehyde-3-phosphate dehydrogenase, peroxidases, pyruvate kinase) and proteins referring to exosomes biogenesis or MVB biogenesis (proteins of ESCRT machinery as Tsg101, Lysobisphosphatidic acid (LBPA) binding protein as Alix), lysosomal markers (LAMP1/2), signalling proteins (kinases, 14-3-3, GTPase Hras, RhoA, RAP1B, Guanine nucleotidebinding protein subunits $-G$ proteins-...), tetraspanins (CD9, CD63, CD81, CD82), proteins involved in transcription and protein synthesis (histones, ribosomal proteins, ubiquitin...), and proteins involved in trafficking and membrane fusion (Annexins, Rab protein family, ARF...).The functional significance of certain proteins is not completely understood. It is worth noting that markers commonly used to characterize a composition of exosomes may have varying distribution between cell types. For instance, the Transferring Receptor (TfR) is absent in exosomes derived from B cells [2] whereas it is present in erythrocyte-derived exosomes [47]. 
In addition to a common set of lipids and proteins, exosomes harbour particular characteristics inherited from the cell type of origin, like CD3 from exosomes derived from $T$ lymphocytes, perforin and granzyme for exosomes from cytotoxic T cells [48]. Intestinal epithelial cells are able to secrete 2 types of exosomes originating from apical and basolateral sides and which display different proteic compositions [6].

\subsection{Lipid content}

It has been characterized that exosomes display a particular lipid organization and composition. A common feature is an enrichment in sphingomyelins ranging from 1.3 times (erythrocytes) to 2.3 times (B lymphocytes) [49]. Cholesterol is enriched only in Blymphocyte-derived exosomes and phosphatidylserine only in dendritic cell-derived exosomes [49], whereas mast cell-derived exosomes are enriched in lysophosphatidylcholine [8]. The phosphatidylcholines and phosphatidylethanolamines present in exosomes are enriched in saturated fatty acids comparatively to the parent cell. Saturated phospholipids are rather rigid molecules at physiological temperature, and subsequently trigger some rigidity of the membrane, which could account for the stability of exosomes invivo. In that respect, exosomes remain functional in lymph nodes for as long as two weeks [50].

It has been shown the presence of lipid raft- like domains in exosome membranes. It has been proposed that lipid micro domains could be involved in the generation of the ILVs since proteins contained in raft-domains (flotilin and stomatin) are incorporated in reticulocytes and B lymphocytes exosomes $[12,51]$, or in concert with tetraspanins $[25,51]$. Tetraspanins are highly enriched in exosomes, and have been localized to raft like microdomains. Indeed, lipid composition analyses have demonstrated that purified exosomes from B cells are enriched in cholesterol, sphingomyelin (SM), glycolipid GM3 [12, 25, 49], glycerophospholipids with long and saturated fatty acyl chains, i.e. lipids that have been found in membrane rafts.

Another important particularity is that exosomes from the mast cell line RBL-2H3 feature an unconventional random transmembrane phospholipid organization [8]. Consistently, proteomic analyses have revealed the presence of a phospholipid scramblase which is involved in mixing the phospholipids between the two membrane leaflets [52]. Exosomes contain phosphatidylserine (PS) which appears to be an essential requirement for their budding within the late endosome.

Among phospholipids, sphingomyelin can be hydrolysed into ceramide by sphingomyelinases. Purified exosomes from Oli-neu cells (mouse, myelinating cells of the central nervous system) are enriched in ceramide, and the release of exosomes was reduced by the inhibition of the neutral sphingomyelin [19]. The lipid composition of these exosomes are similar to lipid rafts, i.e. enriched in cholesterol, containing high amount of sphingolipids (sphingomyelin and hexocylceramide) and lower amount of phosphatidylcholine, an increase in saturated PC at the expense of polyunsaturated species. Ceramides are proposed to induce aggregation of lipid microdomains into larger ones. The cone-shape structure of ceramide molecule might trigger membrane curvature by creating an area difference 
between the membrane leaflets. Ceramides seem to be used for generation of ILV that are not destinated to the lysosomes but secreted as exosomes.

\subsection{RNA content}

An unexpected finding was that exosomes contain RNA. The first evidences demonstrating that exosomes could contain mRNA and microRNA were brought by Valadi and coll. in 2007 [23]. Actually, 1300 different mRNA were identified in exosomes from a mouse mast cell line $\mathrm{MC} / 9$, as many as in BMMC and human mast cell HMC-1 that was estimated to represent approximatively $8 \%$ of the mRNA detected in the donor cells. No less than $\mathbf{2 7 0}$ gene transcripts were exclusively found present in exosomes whereas they were indetectable in the donor cell, and they were related to cellular development, protein synthesis, RNA post-translational modification. Exosomes were also enriched in about 121 microRNA. MicroRNAs are small (22-25 nucleotides in length) noncoding RNAs which interfere with RNA post-transcriptionally by binding to the 3 'untranslated regions of their target mRNAs to degrade it, or suppress, or stimulate translation. Their function are in stem cell differentiation control (let-7), differentiation and organogenesis (miR-1), hematopoiesis (miR-181) and exocytosis and tumorigenesis (miR-17, -18, -19, -20, -19b and -92-1).

An important finding is that these mRNA and microRNA are functional since they could be in -vitro transcribed into mouse protein (from mast cells exosomes $\mathrm{MC} / 9$ ) in human mast cells HMC-1. Thus, exosomes appear as vehicle for RNA probably intervening both in the microenvironment and in the systemic circulation, able to deliver their content and modulate recipient-cell protein production.

It was reported that placental specific miRNAs, released via exosomes, can be exported from the placental trophoblast into maternal circulation where they can be found abundantly. Glioblastoma tumour cells release exosomes containing mRNA and microRNA, translated into brain microvascular endothelial recipient cells, after internalization [53]. Exosomes also contain ESCRT-II subunits able to specifically bind mRNA. It was also discovered the presence of microRNAs within exosomes isolated from fresh and frozen glandular and whole human saliva [54] Recently, exosomes from human saliva have been characterized for their mRNA content and 509 mRNA were found. In vitro, exosomes were able to transfer their genetic information to human oral keratinocytes modifying gene expression. In EBV-transformed lymphoblastoid $B$ cells ( $L C L)$, the presence of miRNA has been detected in purified exosomes among which BHRF1 and BART EBV-miRNAs that are abundantly represented in EBVassociated tumors. LCL-exosomes were capable of transfer EBV-miRNA in recipient monocyte-derived dendric cell, and the EBV-miRNA BHRF1 or EBV-miRNA BART permitted the repression of their specific target mRNAs : CXCL11 or LMP1 respectively. An interesting finding was the discovery of EBV-miRNA in non $B$ cell populations of PBMC recovered from asymptomatic HIV patients, whereas, EBV-DNA was exclusively restricted to $B$ cell population, suggesting miRNA transfer between these cells in vivo. 
All these studies agree with the fact that either specific microRNAs, proteins and lipids found in exosomes could represent a basis on which can be developed future biomarkers of the diagnosis and prognosis of diverse pathologies.

\section{Exosome as biomarkers of pathologies}

It has been observed that biological fluids in the body carry exosomes, and because of their specific protein, lipid and RNA content, biomarkers associated with these exosomes may be useful for early detection of diseases.

Exosomes in urine are useful markers of kidney diseases. It is possible to detect the mRNA of two molecules known to be overexpressed in prostate cancer, PCA3 and a specific product resulting from a chromosomal rearrangement, the TMPRSS2:ERG fusion [55]. Also aquaporin 1 and 2 as markers of renal ischemia/reperfusion injury or anti diurectic hormone action respectively [56], have been characterized.

Exosomes released from tumor cells can also be recovered in blood [57]. Analyses of specific microRNA associated with lung tumor, reveal the presence of a set of microRNA in exosomes, purified from serum, and similar to those found in tumor tissues. Therefore microRNA in circulating exosomes are representative of those expressed in the tumor, opening a perspective for early detection of the disease using circulating exosomes as screening tool.

Also exosomes secreted in human saliva contain mRNA that may serve as disease biomarkers. [58], and it has been recovered exosomes from amniotic fluids which could provide potent markers for prenatal diagnosis [59]

Thus exosomes could constitute a source of multiple markers of malignancy that could provide clinically useful information. Moreover, exosomes can be easily recovered in a non invasive manner without the need of tissue. In that respect, specific proteins borne by exosomes derived from tumors constitute potential disease biomarkers [60].

\section{Exosomes as signalosomes in recipient cells: from the cell periphery towards the nucleus}

\section{Exosomes are signalosomes:}

Exosomes feature a high content in protein [49] and proteomic analysis revealed a range of 350-400 different proteins [61] [9]. Exosomes contain peripheral membrane proteins such as MHC I and II [2] integrins, transferring receptors [62], tetraspanins [63] which are active towards downstream signaling pathways in target cells, triggering for instance calcium signaling [64], MAPK activation [65], or NKG2D signalling [66-68]. Exosomes also contain active lipolytic activies such as phospholipases leading to the formation of bioactive lipid mediators (fatty acids and prostaglandins) which can interact with peripheral 
G-protein coupled receptors and nuclear receptors in target cells [9]. Therefore exosomes behave as a vectorized signalling device which appears more efficient than a soluble agonist which can disperse in the outer cell medium.

\section{Molecular interaction of exosomes with recipient cell periphery}

ICAM-1 (intercellular adhesion molecule 1) and LFA-1 (leukocyte function - associated antigen-1) play a role in the capture of the exosomes at the cell surface $[5,69,70]$. It has been shown that ICAM1-bearing exosomes derived from mature DC are captured by binding to LFA1 ( a ligand for ICAM1), on the surface of CD8 ${ }^{+}$DCs $[70]$ and activated T cells $[69,70]$. Exosomes express various adhesion molecules such as VLA-4 (reticulocytes and B cell exosomes), $\alpha M$ integrin, $\beta 2$ integrin (dendritic cell exosomes), which potentially mediate adhesive interactions to a multitude of ligands, including cell surfaces and/or to extracellular matrix components.

A diminution of exosome uptake by dendritic cells was observed in-vivo by inhibition of $\alpha v \beta 3$ integrins, CD11a and its ligands CD54, antibodies directed against the tetraspanins CD9 and CD81, or a soluble analogue of phosphatidylserine (PS) [71]. Thus exosomes adhesion to the recipient cell membrane are mediated through lipids (PS) and ligand-receptor interactions. An implication of the Milk fat globule-Epidermal Growth factor VIII protein (MFGE8 or lactadherin) was also established [71]. The amino-terminal domain of MFGE8 binds to $\alpha v \beta 3$ and $\alpha v \beta 5$ integrins, and promotes their phagocytosis by macrophages [72].

CD91 has been identified as the common receptor for several heat shock proteins (including Hsp90, Hsp70, , Hsp60...) and as an endocytic receptor that internalizes antigenic proteins or peptides chaperoned by Hsp. Treating a target cell with an anti-CD91 antibody or the RAP peptide (a ligand for CD91 receptor) decreases the functional effect of mast cellderived exosomes [73].

Exosome phospholipids are randomly distributed between the two membrane leaflets, but because of their membrane curvature, about two third of the phospholipids are exposed to the outer layer of their membrane [8]. This accounts for an apparent enrichment of PS in the outer layer. Thus, multiple PS binding proteins on recipient cell are susceptible to link exosomes, and this includes several classes of scavenger receptors, integrins, complement receptors and CD14[5] and PS receptors [74]. Two PS receptors have been described, i.e TIM1 and TIM-4 (T-cell Immunoglobulin - Mucin domain containing molecules 4) which recognizes PS via the immunoglobulin domain, but it is TIM-4 which preferentially binds exosomes and mediates their engulfment [75].

TIM-1 is expressed on the surface of activated lymphocytes (Th2 cells) and TIM-4 is expressed in phagocytes (macrophages) and various tissues such as the spleen, thymus, lymph nodes and salivary glands. PS receptor play a key role in exosome-mediated cholesterol accumulation in human monocytes and related cell lines such as THP-1 cells [74]. 


\section{Exosome internalization}

Exosome internalization by target cells appears to depend upon the type of recipient cells. During T cell activation, LFA-1 present on the cell periphery undergoes a transient conformational change resulting in a high-affinity conformation against its receptor ICAM-1 borne by exosomes. In that case, exosomes can be presented by mouse dendritic cells without being internalized and processed [70].

However evidences have also been reported for internalisation of exosomes by recipient cells ([23],[71], [53]) and the extent of exosome internalization might depend upon the phagocytic capabilities of the recipient cell [76]. In any cases, since miRNA vehiculated by exosomes are able to modulate genes in a target cell [23], this indicates that exosomes are able to deliver their content into the recipient cells. Delivery of exosome content can occur either by fusion between exosome and the plasma membrane [77], internalization of exosome with subsequent opening of the vesicule inside target cell [78], or internalization and degradation of the exosome membrane inside the cell [35].

Internalization of exosome content by its fusion with the plasma membrane might be limited to acidic $\mathrm{pH}$ conditions such as that found inside a tumor [77]. Membrane fusion requires similar fluidity between the two membranes supposed to fuse. Exosome and plasma membrane display the same fluidity at $\mathrm{pH} 5$ [8], but not at neutral $\mathrm{pH}$ which makes the membrane more rigid, therefore precluding a fusion with another membrane [9]. Noteworthy pH value of the MVB is about 5, and fusion of the ILVs present in MVB (Fig1) back to the MVB limiting membrane has been reported to occur [79].

Internalization of exosomes appears as a general process. It has been argued however that many works reporting internalization actually monitored the fate of exosome aggregates, since exosomes spontaneously aggregate in-vitro. However exosomes exit the cell as aggregates in activated T cells [80], and natural transfer of exosomes between two different type of cells in co-culture by macropinocytosis has been recently reported [81]. In hematopoietic-derived cell lines (K562, MT4) exosomes were shown to enter cells via phagocytosis and not by macropinocytosis or by internalization pathways involving caveolae or clathrin-coated vesicles [76]. In the mast cell line RBL-2H3, exosome internalization occurs after a lag time of about 15-20 min, and thereafter accumulated in late endosomes (MVB) of target cells following a sustained pulse [9]. This observation indicates that the MVB compartment features a dual function of being a producer of exosomes but also a receiver of exogenous exosomes. In each case a different subset of MVB would be involved, since exosome release is sectorized in the cell [80] and appearsnot involve all the MVBs. In that respect two distincts MVB pathways, one for lysosomal targeting and the other for exosome secretion have been characterized [17].

One can conceive that exogenous exosomes being internalized into a resting cell could in turn be released upon activation of this cell and reach another recipient cells. Therefore exosomes issued from an initial producer cell could propagate from cells to cells. This "jumping pathway" could provide a way for exosomes to cross the thickness of a tissue. This could explain how viruses like HIV can cross the blood-brain barrier. HIV and exosome feature a similar size and share some protein content [82]; and HIV virions are partly 
generated into the MVB [82]. In addition recent developments demonstrated that HIV enters the endocytic track of target cells for infection [83].

Internalization of exosomes could have another functional involvement, i.e to deliver nuclear receptor modulators. It has been recently shown that exosomes carry bioactive lipidic mediators such as the PPAR $\gamma$ nuclear receptor modulator 15d-PGJ2 [9]. Whether this exosome-mediated transport of modulator is further able to activate PPAR $\gamma$-dependent gene transcriptionremains to be established, but if so, it would make exosomes the first adressing mechanism operating from the MVB of a cell directly to the nucleus of another cell.

\section{Exosomes in the body: an integrated network involved in multiple pathophysiologies}

\section{Beneficial effects of exosomes in health}

\subsection{Reticulocyte differentiation.}

Although there is a growing interest in exosome secretion, which now appears as a general process from different type of cells, it has to be borne in mind that they were first described about 30 years ago by $\operatorname{Dr}$ Rose Johnstone working on reticulocyte maturation, as a mechanism to release obsolete proteins [1]. Reticulocyte differentiation is a permanent biological process in the body and about $1 \%$ of the total red blood cells ( i.e $230 \times 10^{9}$ cells) is renewed every day from reticulocytes. It has been estimated that about $2 \times 10^{12}$ exosome particles from reticulocytes would be released per day in the blood stream ${ }^{(b)}$. Exosomes exhibit a higher protein /phospholipid ratio than parental cells [49] i.e they are enriched in proteins. Considering that $6 \times 10^{5}$ exosome particles contain $1 \mathrm{mg}$ of protein [9], exosome accumulation in the body from reticulocyte maturation would constitute a mass of 3 kilos a day. Therefore an efficient exosome clerarance mechanism appears necessary to maintain a steady-state content of these vesicles in the body. So far, clearance of reticulocyte exosomes has been shown to involve a similar mechanism to that involved in elimination of apoptotic cells and regulated by the activity of a calcium-independent phospholipase A2 (iPLA2)[84]. Exosomes are enriched in a phospholipid, namely the lyso-phosphatidylcholine (LPC) [8] which can originate from an iPLA2 activity, which itself is activated by reactive oxygen species [84]. LPC constitutes a "eat-me signal" for apoptotic cells, allowing the clearance of the reticulocyte exosomes [84]. Also exosome surface proteins such as galectin-5 has been shown to modulate reticulocyte exosomes uptake by macrophages [85]. Alltogether these data indicate a very high turn-over of exosomes in the body.

(b) Communicated at the $1^{\text {rst }}$ Exosome Workshop, Mc Gill University, Montreal , 2005 


\subsection{Stimulation of immunocompetent cells.}

About ten years later after the characterization of reticulocyte-derived exosomes, the observation by Dr Graça Raposo that B lymphocytes secrete antigen presenting vesicles brought the exosomes in the field of immunology [2]. The observation that dendritic cellderived exosomes bear molecules involved in direct T cell activation, including MHC class I and II complex, costimulatory molecules such as CD40, CD80, CD86, Heat Shock Proteins, conferring them a role in antigen presentation has triggered numerous investigations to assess exosomes potency to modulate the immune response. A few years later, exosomes were brought in the field of cancer treatment by immunotherapy, since exosomes derived from murine bone marrow dendritic cells loaded with tumor peptides display antitumor activity in-vivo, both towards the murine mastocytoma P815 and the murine mammary carcinoma TS/A tumor models [86]. It was also observed that $T$ cells were primed by exosomes in an antigen specific manner and an antitumor activity mediated by cytotoxic lymphocytes was revealed in treated animals [87].

Moreover, exosomes can be viewed as an amplification process for dendritic cellmediated T cytotoxic responses, based on the fact that $2.10^{9}$ exosomal MHC class I molecules can be released by $10^{4}-10^{5}$ immature DCs in vitro in $24 \mathrm{~h}$ [88]. Dendritic cells derived exosomes were also shown to regulate NK cells functions, such as inflammatory cytokines (TNF? and IFN?) production, via the protein HLA-B associated transcript-3 (BAT3) expressed on their surface [89]. This intracellular protein which is involved in DNA damageinduced apoptosis represents the ligand for a natural cytotoxicity receptor expressed on NK cell, the NKp30, involved in direct cytotoxicity against tumor cells.

The first clinical trials using immature DC derived exosomes (Dex) as a cell free vaccine against advanced melanoma and lung cancer bearing patients failed to detect vaccine specific $\mathrm{T}$ cell responses while observing potent Dex-related NK cell activation [90], suggesting that Dex can also stimulate the innate immune response.

Mast cell exosomes also participate in the immune response since they have the capacity to stimulate B and T cell proliferation and cytokines production such as IL-2 and IFN $\gamma$ [91]. It was shown that peptide-loaded exosomes from mast cells elicit an efficient antibodies production in-vivo following a subcutaneous injection in mice. Mast cell-derived exosomes can trigger phenotypic and functional maturation of dendritic cells.

\subsection{Exosomes as therapeutic tool}

Following injection in patients of Dex loaded with antigenic peptides from human melanoma during the phase I clinical trials, it was observed an increase of NK cells number and that NKG2D expression was restored in NK cells and CD8 T cells in some patients[92]. Given the requirement of IL-2 and transpresentation of IL-15 to IL-15R $\alpha$ for NK cell survival, homeostasis and proliferation, it was reported that Dex can directly trigger NK cell proliferation involving IL-15R $\alpha$. NKG2D is an activating receptor with a $C$ type lectin-like domain, expressed on all natural killer (NK) cells, TCR $\gamma \delta$ and $C D 8^{+}$TCR $\alpha \beta$ T cells. NKG2D can either costimulate the activation of naïve $T$ cells or trigger cytotoxicity in the absence of $T$ cell receptor (TCR) ligation. 
A Phase II clinical trial was also evaluated on patients bearing inoperable stage III/IV non-small cell lung cancer (NSCLC) after stabilization or regression with cycles of conventionnal chemotherapy. Dex vaccination with exosomes bearing IL-15R $\alpha$ and NKG2D are used in association with cyclophosphamide treatments after chemotherapy [90]. In this approach, cyclophosphamide allows the inhibition of regulatory $\mathrm{T}$ lymphocytes (Treg), facilitates $T$ cell priming mediated by Dex, and permits to restore T and NK cell functions in end stage patients.

Therefore exosomes appear as a therapeutic tool able to trigger an immune response against a tumor. In the above-mentionned clinical trials, tumors displayed well-defined antigens, which is not the case of most of tumors. In these assays, antigenic tumor peptides were acid-pulsed directly on exosomal MHC I, or loaded by endocytosis on MHC II of exosomes at the stage of ILV inside the MVB of dendritic cells [92]. Fluid-phase endocytosis also allows to load exogenous proteins inside the ILV, which illustrates a way to load exosomes which tumor cell extracts when specific tumor antigens are not known. Exosomemediated immunotherapy could be refered as a cellular type of therapy since they are a biological product. However they are more convenient to handle than a cell, since they are stable vesicles which keep their biological activities for at least two years at $-80^{\circ} \mathrm{C}$. After storage there is no need to expand them, and they can be used directly, either alone or in combination with other pharmacological agent. In addition, they maintain the antigen presentation within a lymph node two times longer than an antigen presenting cell, which indicates they potentiate the immune response [50]. The only limitations are that they must be autologous, prepared and used in the same patient, and that the yield of tumor antigenloaded exosomes prepared from dendritic cells features large variations between individuals.

\section{Diversion of the exosome pathway in pathologies.}

\subsection{Tumor promoter activity.}

Opposite to the potent immune stimulatory properties of exosomes issued from immunocompetent cells, many studies have demonstrated that tumor cell-derived exosomes display detrimental effects on immune response against tumors, leading to suppressive pathways hampering immune defenses in patients [91]. Consequently, tumor exosomes may contribute to tumor growth and to participate in tumor evasion.

Exosomes from tumors cells harbor NKG2D ligand such as MICA*008 or ULBP3 proteins, which suppress Natural Killer cell toxicity [93]. Ligation of NKG2D with its ligands led to a down regulation of NKG2D expression. Thus, tumor exosomes bearing NKG2D ligands can act on effector cells to down regulate NKG2D, impairing part of the immune response.

In addition to NKG2D ligands, it was shown that tumor exosomes carry and express growth factors such as TGF $\beta 1$. Membrane-bound transforming growth factor- $\beta 1$ on these vesicles displays the same function as NKG2D ligands i.e. the down regulation of surface 
NKG2D expression by NK cells and $C D 8^{+} T$ cells following a direct interaction between exosomes and cells.

Also exosomes secreted from tumor cell lines or tumor cells coming from patients can induce T cell apoptosis in-vitro through the expression of death ligands such as CD95 ligand (FASL) and TRAIL [35, 66].

Tumor-derived exosomes promote other immune escape mechanisms by triggering myeloid suppressive cells proliferation (MDSC). These cells are found in large number in lymphoid organs, blood and tumor tissues in cancer patients and are immature myeloid cells. These cells express myeloid marker stimulatory molecules (CD14 and CD11b) and are devoid of co-stimulatory molecules (HLA DR, CD80, CD86). They spontaneously secret TGF $\beta$ and have suppressive activity on activated $\mathrm{T}$ lymphocytes since they are able to inhibit $\mathrm{T}$ cells proliferation and cytolytic functions [94]. It was shown that melanoma and colorectal carcinoma-derived exosomes altered the monocyte differentiation into dendritic cells, leading to the generation of myeloid suppressive cells [94]. Furthermore, it was demonstrated that MDSC-mediated promotion of tumor progression was dependent on TGF- $\beta$ present on exosomes, but also depended upon the lipidic mediator prostaglandin E2 (PGE2) transported by tumor exosomes [95].

The angiogenic properties displayed by tumor exosomes has also been documented. In a model of endothelial spheroid generated in matrigel with an endothelial cell line capable of spontaneously aggregates in spheroids, it was demonstrated that melanoma (B16) exosomes could promote angiogenesis, raising the communication network between cells. [96]. Given that hypoxic cells are thought to acquire a metastatic character, a quantitative proteomic of carcinoma exosomes under hypoxia have been performed and revealed the presence of proteins involved in metastasis and angiogenesis [97]. It was observed that mRNA found in glioblastoma exosomes could be translated in recipient cells such as endothelial cells and revealed the presence of proteins with angiogenic properties, stimulating tubule formation [53]. Also transcripts related to cell cycle were evidenced in colorectal exosomes that was concordant with their capacity to modulate endothelial cells cycle and proliferation, underlying their involvement in angiogenesis [98].

\subsection{Spreading pathogens}

Various pathogens are able to divert the "exosome pathway" such as the HIV virus. When infected, dendritic cells, monocytes, macrophages and lymphocytes can produce both exosomes and HIV virions [82] with similar density, which require separation by immunocapture or appropriate gradient density separation. Also exosomes compete with HIV for the virus entry in endocytic compartment [83]. About $10 \%$ proteins are identical between HIV virions and exosomes issued from the same cell type [82].

Mycobacterium avium is a major opportunistic pathogen in HIV-positive individuals and is responsible for increased morbidity and mortality in AIDS patients. M. avium express glycopeptidolipids (GLPs) as a major cell wall constituent. M avium-infected macrophages (J774) release exosomes containing GLPs. Thus, they permit the transfer of GLPs from infected to uninfected macrophages. Infected macrophages release exosomes that incorporate bacterial molecules PAMPs (Pathogen-Associated Molecular Pattern) which acts 
as TLR ligands and lead to uninfected macrophages activation. Thus exosomes from infected macrophages can stimulate a proinflammatory response (TNF- $\alpha$ and RANTES secretion) in resting macrophages [99]. Interestingly, exosomes loaded with curcumin, a natural polyphenol, display anti-inflammatory properties [100].

In central nervous system, proteins such as Prion responsible of Creutzfeldt-Jacob disease or Amyeloid Precursor Protein (APP) involved in Alzheimer disease are carried by exosomes. Prion disease leads to the accumulation in the central nervous system of the abnormally folded prion protein (PrP) scrapie (PrPsc). Alzheimer's disease is characterized by a continuous loss of neurons and the extracellular accumulation of insoluble amyloid fibrils of APP in the brain

Therefore the normal exosome biogenesis pathway can be "hijacked" by viruses and bacteria in cells, and also by abnormal proteins responsible of diseases of the central nervous system.

\section{E. Pharmacology of exosomes: the future challenge}

The qualitative and quantitative modulation of exosomes produced by cells appears as a challenge to intervene in their biological effect and fight diseases. To favor the immune response against tumors one can conceive to enhance the production of immunocompetent exosomes and to decrease those released from tumor cells. In the clinical trials undertaken so far, the amount of immunocompetent exosomes produced "ex-vivo" by dendritic cells issued from patients bearing melanoma was highly variable and was a limiting step in that immunotherapy approach. Therefore understanding in depth the biogenesis and release mechanisms of exosomes in cells is essential. It has been shown that some lipid mediators could enhance their production $[45,101]$. On the reverse, decreasing the amount of tumor exosomes released by modifying the $\mathrm{pH}$ of MVB with amiloride enhances the efficacy of chemotherapeutic agents [102].

Exosomes carry a variety of receptor ligands towards the close environment as well as to distant cells. Exosomes from tumors cells harbor NKG2D ligand such as MICA*008 or ULBP3 proteins, which suppress Natural Killer cell toxicity [93]. They are also enriched in an immunosuppressive lipid, the prostaglandin E2 (PGE2) [9] which interacts with a family of four GPCR (EP1 to EP4). PGE2 provides a coordinated regulation of tumor immunosupression by inhibiting NK cell and T-CD8 cell functions, dendritic cell maturation, and by triggering Tregulator and Myeloid Derived Suppressor Cells (MDSC) expansion. On the reverse, exosomes carry the ligand (prostaglandin 15d-PGJ2) of the nuclear receptor PPAR $\gamma$, whose engagement triggers tumor growth inhibition by antiproliferative and proapoptotic effects, anti-angiogenic properties and by promoting cell differentiation [103].It remains to establish that exosome-transported 15d-PGJ2 can interact with PPAR $\gamma$, as recently suggested [9]. Also it was reported that exosomes contain a coactivator (the phosphatidate phosphatase PAP1) of the nuclear PPAR $\alpha$ receptor [9]. Exosomes also transport high concentrations of polyunsaturated fatty acids such as arachidonic acid [9], which is the precursor of prostaglandins, and which can potentially be addressed to nuclear 
receptors such as LXR. Therefore exosomes could signal between the MVB of a donor cell and the nucleus of a target cell, providing that nuclear receptor activation by exosomebound ligands be established. Since 15d-PGJ2 and PGE2 display opposite effects on tumor growth, the balance between these two ligands could define exosome function in a tumor microenvironment. Results on prostaglandins were obtained on exosomes released from a rat basophil leukemia cell line (RBL-2H3)[9], and it is required to screen more tumor-derived exosomes to assess whether the prostaglandin profile of exosomes could account for tumorprogression in-vivo. It is worth to note that incubating tumor exosomes with anti-PGE2 antibody decreases their effect on myeloid-derived suppressor cell expansion [95].

Exosomes released in the circulation can interact with distant cells by means of receptor adhesion such as ICAM1 which interacts with LFA1 on target cells, or the Milk Fat Globulin MFGE8 which links phosphatidylserines (PS) on exosomes with integrins of targets cells. PS are also the ligand of Tim1 and Tim4, receptors involved in internalization of exosomes. Exosome uptake can also be mediated by CD91, a common receptor of heat shock proteins (Hsp 70, Hsp 90) borne by exosomes. Therefore one can conceive that distant supply of immunosuppressive molecules such as PGE2 by tumor exosomes could prepare a premetastatic niche. Inhibiting the release of tumor exosomes would be a therapeutic strategy to prevent tumor growth and metastasis. In that respect, decreasing tumor exosome production using dimethyl amiloride, a drug used to treat high blood pressure, enhanced the in-vivo antitumor efficacy of the chemotherapeutic drug cyclophosphamide [102]. This observation suggests that sodium channel blockers could constitute a pharmacological class of agents to block tumor exosome release. In that respect it would be worth to screen the effect of various anticancer drugs on their ability to inhibit tumor exosome release. However a general treatment avoiding exosome release in cancer patients might have side effects, since the release of immunocompetent exosomes which can enhance tumor recognition by the immune system [86] would be inhibited as well. Therefore an optimal treatment would require differential therapeutic targets between tumor and immunocompetent cells. One can consider that an efficient cancer treatment would trigger an enhanced immunocompetent exosome biogenesis and/or release, while inhibiting tumor exosome production. Therefore a screening of drugs used to cure cancers regarding their effect on the ratio between immunocompetent ( $M H C$ bearing)-exosomes and tumor (immunosuppressive-containing molecules)-exosomes, both in-vitro in cell culture and invivo in serum from patients, would bring new perspectives on the efficacy of the various treatments.

Actually, increasing the production of immunocompetent exosomes loaded with tumor peptide ligands on their MHC molecules would provide a higher engagement of the immune system towards tumors [86]. New generation of dendritic cell-derived exosomes for cancer immunotherapy have been devised [90] and clinical trials are ongoing. The biogenesis of exosomes from dendritic cells was reported earlier to be variable between patients and could affect the issue of the therapy [92]. Thus enhancing exosome production would improved the process, and this could be achieved by manipulating the biosynthesis of a fusogenic lipid, namely phosphatidic acid, by overexpression of the lipolytic enzyme phospholipase D (PLD2), which increases by two times exosome amount [101]. Therefore 
PLD2 appears also as a pharmacological target to enhance exosome production (although it would not apply in case of viral infection; see Fig 3 ) and its role should be assessed by newly developped specific inhibitors [104].

The heat shock protein Hsp 70 is also involved in antigen presentation by enhancing peptide transfer on MHCs, and overexpression of Hsp 70 in tumor cells reverses the deleterious effects of tumor exosomes [105]. Thus the phenotypic modification of exosomes represents an alternative strategy to switch tumorogenic exosomes into anti-tumor ones. This can be achieved by treating tumor cells with recently characterized new lipidic molecules, namely alkylaminooxysterols [106], able to enhance the Hsp70 expression in exosomes from tumor cells, thus leading to tumor-derived exosomes with anti-tumor activities [107].

Modification of the quality of exosomes, i.e the panel of protein and lipids or $\mathrm{mRNA} /$ miRNA they contain, appears as a therapeutic strategy. Since exosome formation occurs in a well-defined cellular compartment, the MVB, modification of the molecular content of this compartment constitutes a possibility to modify also exosomes. Interestingly MVB is accessible from outside of the cell via the endocytic track. Chanelling therapeutics to the MVB compartment might be an innovative strategy.

\section{Conclusion}

Generation of vesicles has been for long considered only $i$ / for cells undertaking activation states, such as hematopoietic cells involved in host defense against pathogens and which release plasma membrane-derived microvesicles also called ectosomes (generally with higher size than exosomes), and $i$ /for cells in the course of apoptotic processes, and which release microparticles containing processed DNA. Among this panel of vesicles, exosomes feature a unique place. They are released by viable cells, whether they are quiescent or activated, from an intracellular compartment, and display a panel of protein which is distinct from the other types of cell-derived vesicles. In addition, they have been evidenced to carry functional mRNAs/miRNAs, and although the same observation has been claimed for microvesicles, many of the microvesicle preparations reported in the literature appeared to contain also exosomes. Well-defined protocols for exosome purification have been established [108]. Since one can assume that all cell types release exosomes, a large diversity of proteins are released and account for a large part of the secretome, i.e the pool of proteins secreted by cells (http://spd.cbi.pku.edu.cn). Specific markers of exosomes are tentatively summarized by the ExoCarta database (http://exocarta.ludwig.edu.au).

In summary, exosomes contain protein and lipid determinants which allow them to interact with target cells, avoiding their dilution in the intercellular space. This "vectorized" signaling appears more efficient than soluble agonists that can be diluted in the outside cellular medium. They provide a unique mechanism to deliver mRNAs to another cells [23], as well as nuclear receptor agonists [9]. Exosomes appear as a "multisignalling device", able to signal to target cells, either at the cell periphery or to bring information to the cytosol and the translation machinery, and possibly to the nucleus as well. They appear as a promising 
tool for therapeutic improvements. Because of their involvement in both positive and negative aspects of health, researches related to exosomes will keep increasing in the future ${ }^{(c)}$.

(c) The second workshop on exosomes was held in January 2011 (Institut Curie, Paris) and gathered more than 200 hundred people, i.e ten times more than at the 1rst workshop (2005, Montreal, Canada).

\section{References}

We apologize to authors whose work has not been cited or indirectly cited because of space constraints.

[1] Johnstone RM, Adam M, Hammond JR, Orr L, Turbide C. Vesicle formation during reticulocyte maturation. Association of plasma membrane activities with released vesicles (exosomes). J Biol Chem 1987;262:9412-20.

[2] Raposo G, Nijman HW, Stoorvogel W, Liejendekker R, Harding CV, Melief CJ, et al. B lymphocytes secrete antigen-presenting vesicles. J Exp Med 1996;183:1161-72.

[3] Eken C, Gasser O, Zenhaeusern G, Oehri I, Hess C, Schifferli JA. Polymorphonuclear neutrophil-derived ectosomes interfere with the maturation of monocyte-derived dendritic cells. J Immunol 2008;180:817-24.

[4] Thery C, Boussac M, Veron P, Ricciardi-Castagnoli P, Raposo G, Garin J, et al. Proteomic analysis of dendritic cell-derived exosomes: a secreted subcellular compartment distinct from apoptotic vesicles. J Immunol 2001;166:7309-18.

[5] Simons M, Raposo G. Exosomes--vesicular carriers for intercellular communication. Curr Opin Cell Biol 2009;21:575-81.

[6] van Niel G, Raposo G, Candalh C, Boussac M, Hershberg R, Cerf-Bensussan N, et al. Intestinal epithelial cells secrete exosome-like vesicles. Gastroenterology 2001;121:337-49.

[7] Keller S, Sanderson MP, Stoeck A, Altevogt P. Exosomes: from biogenesis and secretion to biological function. Immunol Lett 2006;107:102-8.

[8] Laulagnier K, Motta C, Hamdi S, Roy S, Fauvelle F, Pageaux JF, et al. Mast cell- and dendritic cell-derived exosomes display a specific lipid composition and an unusual membrane organization. Biochem J 2004;380:161-71.

[9] Subra C, Grand D, Laulagnier K, Stella A, Lambeau G, Paillasse M, et al. Exosomes account for vesicle-mediated transcellular transport of activatable phospholipases and prostaglandins. J Lipid Res 2010;51:2105-20. 
[10] Stoorvogel W, Kleijmeer MJ, Geuze HJ, Raposo G. The biogenesis and functions of exosomes. Traffic 2002;3:321-30.

[11] Katzmann DJ, Odorizzi G, Emr SD. Receptor downregulation and multivesicularbody sorting. Nat Rev Mol Cell Biol 2002;3:893-905.

[12] Fevrier B, Raposo G. Exosomes: endosomal-derived vesicles shipping extracellular messages. Curr Opin Cell Biol 2004;16:415-21.

[13] Raiborg C, Rusten TE, Stenmark H. Protein sorting into multivesicular endosomes. Curr Opin Cell Biol 2003;15:446-55.

[14] Matsuo H, Chevallier J, Mayran N, Le Blanc I, Ferguson C, Faure J, et al. Role of LBPA and Alix in multivesicular liposome formation and endosome organization. Science 2004;303:531-4.

[15] Kobayashi T, Stang E, Fang KS, de Moerloose P, Parton RG, Gruenberg J. A lipid associated with the antiphospholipid syndrome regulates endosome structure and function. Nature 1998;392:193-7.

[16] Marsh M, van Meer G. Cell biology. No ESCRTs for exosomes. Science 2008;319:1191-2.

[17] Buschow SI, Nolte-'t Hoen EN, van Niel G, Pols MS, ten Broeke T, Lauwen M, et al. MHC II in dendritic cells is targeted to lysosomes or T cell-induced exosomes via distinct multivesicular body pathways. Traffic 2009;10:1528-42.

[18] Hemler ME. Specific tetraspanin functions. J Cell Biol 2001;155:1103-7.

[19] Trajkovic K, Hsu C, Chiantia S, Rajendran L, Wenzel D, Wieland F, et al. Ceramide triggers budding of exosome vesicles into multivesicular endosomes. Science 2008;319:1244-7.

[20] Blanc L, Vidal M. Reticulocyte membrane remodeling: contribution of the exosome pathway. Curr Opin Hematol 2010 17:177-83.

[21] Fang Y, Wu N, Gan X, Yan W, Morrell JC, Gould SJ. Higher-order oligomerization targets plasma membrane proteins and HIV gag to exosomes. PLoS Biol 2007;5:e158.

[22] Sahu R, Kaushik S, Clement CC, Cannizzo ES, Scharf B, Follenzi A, et al. Microautophagy of cytosolic proteins by late endosomes. Dev Cell 20:131-9.

[23] Valadi H, Ekstrom K, Bossios A, Sjostrand M, Lee JJ, Lotvall JO. Exosome-mediated transfer of mRNAs and microRNAs is a novel mechanism of genetic exchange between cells. Nat Cell Biol 2007;9:654-9.

[24] Gibbings DJ, Ciaudo C, Erhardt M, Voinnet O. Multivesicular bodies associate with components of miRNA effector complexes and modulate miRNA activity. Nat Cell Biol 2009;11:1143-9.

[25] Wubbolts R, Leckie RS, Veenhuizen PT, Schwarzmann G, Mobius W, Hoernschemeyer J, et al. Proteomic and biochemical analyses of human B cell-derived exosomes. Potential implications for their function and multivesicular body formation. J Biol Chem 2003;278:10963-72.

[26] Kobayashi T, Beuchat MH, Chevallier J, Makino A, Mayran N, Escola JM, et al. Separation and characterization of late endosomal membrane domains. J Biol Chem 2002;277:32157-64.

[27] Falguieres T, Luyet PP, Gruenberg J. Molecular assemblies and membrane domains in multivesicular endosome dynamics. Exp Cell Res 2009;315:1567-73.

[28] Bouvier J, Zemski Berry KA, Hullin-Matsuda F, Makino A, Michaud S, Geloen A, et al. Selective decrease of bis(monoacylglycero)phosphate content in macrophages by high supplementation with docosahexaenoic acid. J Lipid Res 2009;50:243-55.

[29] Chevallier J, Chamoun Z, Jiang G, Prestwich G, Sakai N, Matile S, et al. Lysobisphosphatidic acid controls endosomal cholesterol levels. J Biol Chem 2008;283:27871-80.

[30] Abdul-Hammed M, Breiden B, Adebayo MA, Babalola JO, Schwarzmann G, Sandhoff K. Role of endosomal membrane lipids and NPC2 in cholesterol transfer and membrane fusion. J Lipid Res 2010 51:1747-60. 
[31] Paillasse MR, de Medina P, Amouroux G, Mhamdi L, Poirot M, Silvente-Poirot S. Signaling through cholesterol esterification: a new pathway for the cholecystokinin 2 receptor involved in cell growth and invasion. J Lipid Res 2009;50:2203-11.

[32] de Medina P, Paillasse MR, Segala G, Poirot M, Silvente-Poirot S. Identification and pharmacological characterization of cholesterol-5,6-epoxide hydrolase as a target for tamoxifen and AEBS ligands. Proc Natl Acad Sci U S A 2010; 107:13520-5.

[33] de Medina P, Payre B, Boubekeur N, Bertrand-Michel J, Terce F, Silvente-Poirot S, et al. Ligands of the antiestrogen-binding site induce active cell death and autophagy in human breast cancer cells through the modulation of cholesterol metabolism. Cell Death Differ 2009;16:1372-84.

[34] Wang P, Zhang Y, Li H, Chieu HK, Munn AL, Yang H. AAA ATPases regulate membrane association of yeast oxysterol binding proteins and sterol metabolism. EMBO J 2005;24:2989-99.

[35] Thery C, Ostrowski M, Segura E. Membrane vesicles as conveyors of immune responses. Nat Rev Immunol 2009;9:581-93.

[36] Ponnambalam S, Baldwin SA. Constitutive protein secretion from the trans-Golgi network to the plasma membrane. Mol Membr Biol 2003;20:129-39.

[37] Savina A, Furlan M, Vidal M, Colombo MI. Exosome release is regulated by a calcium-dependent mechanism in K562 cells. J Biol Chem 2003;278:20083-90.

[38] Loomis RJ, Holmes DA, Elms A, Solski PA, Der CJ, Su L. Citron kinase, a RhoA effector, enhances HIV-1 virion production by modulating exocytosis. Traffic 2006; 7:1643-53.

[39] Savina A, Fader CM, Damiani MT, Colombo MI. Rab11 promotes docking and fusion of multivesicular bodies in a calcium-dependent manner. Traffic 2005;6:131-43.

[40] Ostrowski M, Carmo NB, Krumeich S, Fanget I, Raposo G, Savina A, et al. Rab27a and Rab27b control different steps of the exosome secretion pathway. Nat Cell Biol 2010; 12:19-30; sup pp 1-13.

[41] Muntasell A, Berger AC, Roche PA. T cell-induced secretion of MHC class II-peptide complexes on B cell exosomes. Embo J 2007;26:4263-72.

[42] Thery C, Regnault A, Garin J, Wolfers J, Zitvogel L, Ricciardi-Castagnoli P, et al. Molecular characterization of dendritic cell-derived exosomes. Selective accumulation of the heat shock protein hsc73. J Cell Biol 1999;147:599-610.

[43] Qu Y, Dubyak GR. P2X7 receptors regulate multiple types of membrane trafficking responses and non-classical secretion pathways. Purinergic Signal 2009;5:163-73.

[44] Lespagnol A, Duflaut D, Beekman C, Blanc L, Fiucci G, Marine JC, et al. Exosome secretion, including the DNA damage-induced p53-dependent secretory pathway, is severely compromised in TSAP6/Steap3-null mice. Cell Death Differ 2008;15:172333.

[45] Alonso R, Rodriguez MC, Pindado J, Merino E, Merida I, Izquierdo M. Diacylglycerol kinase alpha regulates the secretion of lethal exosomes bearing Fas ligand during activation-induced cell death of $\mathrm{T}$ lymphocytes. $\mathrm{J}$ Biol Chem 2005;280:28439-50.

[46] Laulagnier K, Vincent-Schneider H, Hamdi S, Subra C, Lankar D, Record M. Characterization of exosome subpopulations from RBL-2H3 cells using fluorescent lipids. Blood Cells Mol Dis 2005;35:116-21.

[47] Olver C, Vidal M. Proteomic analysis of secreted exosomes. Subcell Biochem 2007;43:99-131.

[48] Blanchard N, Lankar D, Faure F, Regnault A, Dumont C, Raposo G, et al. TCR activation of human $\mathrm{T}$ cells induces the production of exosomes bearing the TCR/CD3/zeta complex. J Immunol 2002;168:3235-41.

[49] Subra C, Laulagnier K, Perret B, Record M. Exosome lipidomics unravels lipid sorting at the level of multivesicular bodies. Biochimie 2007;89:205-12. 
[50] Luketic L, Delanghe J, Sobol PT, Yang P, Frotten E, Mossman KL, et al. Antigen presentation by exosomes released from peptide-pulsed dendritic cells is not suppressed by the presence of active CTL. J Immunol 2007;179:5024-32.

[51] de Gassart A, Geminard C, Fevrier B, Raposo G, Vidal M. Lipid raft-associated protein sorting in exosomes. Blood 2003;102:4336-44.

[52] Pisitkun T, Shen RF, Knepper MA. Identification and proteomic profiling of exosomes in human urine. Proc Natl Acad Sci U S A 2004;101:13368-73.

[53] Skog J, Wurdinger T, van Rijn S, Meijer DH, Gainche L, Sena-Esteves M, et al. Glioblastoma microvesicles transport RNA and proteins that promote tumour growth and provide diagnostic biomarkers. Nat Cell Biol 2008;10:1470-6.

[54] Michael A, Bajracharya SD, Yuen PS, Zhou H, Star RA, Illei GG, et al. Exosomes from human saliva as a source of microRNA biomarkers. Oral Dis 2010;16:34-8.

[55] Nilsson J, Skog J, Nordstrand A, Baranov V, Mincheva-Nilsson L, Breakefield XO, et al. Prostate cancer-derived urine exosomes: a novel approach to biomarkers for prostate cancer. Br J Cancer 2009; 100:1603-7.

[56] Takata K, Matsuzaki T, Tajika Y, Ablimit A, Hasegawa T. Localization and trafficking of aquaporin 2 in the kidney. Histochem Cell Biol 2008;130:197-209.

[57] Koga K, Matsumoto K, Akiyoshi T, Kubo M, Yamanaka N, Tasaki A, et al. Purification, characterization and biological significance of tumor-derived exosomes. Anticancer Res 2005;25:3703-7.

[58] Palanisamy V, Sharma S, Deshpande A, Zhou H, Gimzewski J, Wong DT. Nanostructural and transcriptomic analyses of human saliva derived exosomes. PLoS One 2010;5:e8577.

[59] Keller S, Rupp C, Stoeck A, Runz S, Fogel M, Lugert S, et al. CD24 is a marker of exosomes secreted into urine and amniotic fluid. Kidney Int 2007;72:1095-102.

[60] Logozzi M, De Milito A, Lugini L, Borghi M, Calabro L, Spada M, et al. High levels of exosomes expressing CD63 and caveolin-1 in plasma of melanoma patients. PLoS One 2009;4:e5219.

[61] Mathivanan S, Lim JW, Tauro BJ, Ji H, Moritz RL, Simpson RJ. Proteomics analysis of A33 immunoaffinity-purified exosomes released from the human colon tumor cell line LIM1215 reveals a tissue-specific protein signature. Mol Cell Proteomics 2010;9:197-208.

[62] Kang D, Oh S, Ahn SM, Lee BH, Moon MH. Proteomic analysis of exosomes from human neural stem cells by flow field-flow fractionation and nanoflow liquid chromatography-tandem mass spectrometry. J Proteome Res 2008;7:3475-80.

[63] Nazarenko I, Rana S, Baumann A, McAlear J, Hellwig A, Trendelenburg M, et al. Cell surface tetraspanin Tspan8 contributes to molecular pathways of exosomeinduced endothelial cell activation. Cancer Res 2010;70:1668-78.

[64] Clayton A, Turkes A, Dewitt S, Steadman R, Mason MD, Hallett MB. Adhesion and signaling by B cell-derived exosomes: the role of integrins. Faseb J 2004;18:977-9.

[65] Calzolari A, Raggi C, Deaglio S, Sposi NM, Stafsnes M, Fecchi K, et al. TfR2 localizes in lipid raft domains and is released in exosomes to activate signal transduction along the MAPK pathway. J Cell Sci 2006;119:4486-98.

[66] Clayton A, Tabi Z. Exosomes and the MICA-NKG2D system in cancer. Blood Cells Mol Dis 2005;34:206-13.

[67] Zhang Y, Luo CL, He BC, Zhang JM, Cheng G, Wu XH. Exosomes derived from IL12-anchored renal cancer cells increase induction of specific antitumor response in vitro: a novel vaccine for renal cell carcinoma. Int J Oncol 2010;36:133-40.

[68] Viaud S, Terme M, Flament C, Taieb J, Andre F, Novault S, et al. Dendritic cellderived exosomes promote natural killer cell activation and proliferation: a role for NKG2D ligands and IL-15Ralpha. PLoS One 2009;4:e4942. 
[69] Nolte-'t Hoen EN, Buschow SI, Anderton SM, Stoorvogel W, Wauben MH. Activated T cells recruit exosomes secreted by dendritic cells via LFA-1. Blood 2009;113:197781.

[70] Segura E, Guerin C, Hogg N, Amigorena S, Thery C. CD8+ dendritic cells use LFA-1 to capture MHC-peptide complexes from exosomes in vivo. $\mathrm{J}$ Immunol 2007;179:1489-96.

[71] Morelli AE, Larregina AT, Shufesky WJ, Sullivan ML, Stolz DB, Papworth GD, et al. Endocytosis, intracellular sorting, and processing of exosomes by dendritic cells. Blood 2004;104:3257-66.

[72] Hanayama R, Tanaka M, Miwa K, Shinohara A, Iwamatsu A, Nagata S. Identification of a factor that links apoptotic cells to phagocytes. Nature 2002;417:182-7.

[73] Skokos D, Botros HG, Demeure C, Morin J, Peronet R, Birkenmeier G, et al. Mast cell-derived exosomes induce phenotypic and functional maturation of dendritic cells and elicit specific immune responses in vivo. J Immunol 2003;170:3037-45.

[74] Zakharova L, Svetlova M, Fomina AF. T cell exosomes induce cholesterol accumulation in human monocytes via phosphatidylserine receptor. J Cell Physiol 2007;212:174-81.

[75] Feng D, Zhao WL, Ye YY, Bai XC, Liu RQ, Chang LF, et al. Cellular internalization of exosomes occurs through phagocytosis. Traffic 2010; 11:675-87.

[76] Feng D, Zhao WL, Ye YY, Bai XC, Liu RQ, Chang LF, et al. Cellular Internalization of Exosomes Occurs Through Phagocytosis. Traffic 2010.

[77] Parolini I, Federici C, Raggi C, Lugini L, Palleschi S, De Milito A, et al. Microenvironmental $\mathrm{pH}$ is a key factor for exosome traffic in tumor cells. J Biol Chem 2009;284:34211-22.

[78] Subra C, Jiang G, Prestwich G, Record M. Lipidomics Analysis of Bioactive vesicles on Silica Diol With Efficient Resolution of Polyglycerophospholipids. Anal Biochem 2010;Submitted.

[79] van der Goot FG, Gruenberg J. Intra-endosomal membrane traffic. Trends Cell Biol 2006;16:514-21.

[80] Fomina AF, Deerinck TJ, Ellisman MH, Cahalan MD. Regulation of membrane trafficking and subcellular organization of endocytic compartments revealed with FM1-43 in resting and activated human T cells. Exp Cell Res 2003;291:150-66.

[81] Fitzner D, Schnaars M, van Rossum D, Krishnamoorthy G, Dibaj P, Bakhti M, et al. Selective transfer of exosomes from oligodendrocytes to microglia by macropinocytosis. J Cell Sci 124:447-58.

[82] Chertova E, Chertov O, Coren LV, Roser JD, Trubey CM, Bess JW, Jr., et al. Proteomic and biochemical analysis of purified human immunodeficiency virus type 1 produced from infected monocyte-derived macrophages. J Virol 2006;80:9039-52.

[83] Izquierdo-Useros N, Naranjo-Gomez M, Archer J, Hatch SC, Erkizia I, Blanco J, et al. Capture and transfer of HIV-1 particles by mature dendritic cells converges with the exosome-dissemination pathway. Blood 2009;113:2732-41.

[84] Blanc L, Barres C, Bette-Bobillo P, Vidal M. Reticulocyte-secreted exosomes bind natural IgM antibodies: involvement of a ROS-activatable endosomal phospholipase iPLA2. Blood 2007;110:3407-16.

[85] Blanc L, Vidal M. Reticulocyte membrane remodeling: contribution of the exosome pathway. Curr Opin Hematol 2010.

[86] Zitvogel L, Regnault A, Lozier A, Wolfers J, Flament C, Tenza D, et al. Eradication of established murine tumors using a novel cell-free vaccine: dendritic cell-derived exosomes. Nat Med 1998;4:594-600.

[87] Wolfers J, Lozier A, Raposo G, Regnault A, Thery C, Masurier C, et al. Tumorderived exosomes are a source of shared tumor rejection antigens for CTL crosspriming. Nat Med 2001;7:297-303. 
[88] Andre F, Chaput N, Schartz NE, Flament C, Aubert N, Bernard J, et al. Exosomes as potent cell-free peptide-based vaccine. I. Dendritic cell-derived exosomes transfer functional MHC class I/peptide complexes to dendritic cells. J Immunol 2004;172:2126-36.

[89] Simhadri VR, Reiners KS, Hansen HP, Topolar D, Simhadri VL, Nohroudi K, et al. Dendritic cells release HLA-B-associated transcript-3 positive exosomes to regulate natural killer function. PLoS One 2008;3:e3377.

[90] Viaud S, Thery C, Ploix S, Tursz T, Lapierre V, Lantz O, et al. Dendritic cell-derived exosomes for cancer immunotherapy: what's next? Cancer Res 2010;70:1281-5.

[91] Skokos D, Le Panse S, Villa I, Rousselle JC, Peronet R, David B, et al. Mast celldependent $\mathrm{B}$ and $\mathrm{T}$ lymphocyte activation is mediated by the secretion of immunologically active exosomes. J Immunol 2001;166:868-76.

[92] Escudier B, Dorval T, Chaput N, Andre F, Caby MP, Novault S, et al. Vaccination of metastatic melanoma patients with autologous dendritic cell (DC) derived-exosomes: results of thefirst phase I clinical trial. J Transl Med 2005;3:10.

[93] Ashiru O, Boutet P, Fernandez-Messina L, Aguera-Gonzalez S, Skepper JN, ValesGomez M, et al. Natural killer cell cytotoxicity is suppressed by exposure to the human NKG2D ligand MICA*008 that is shed by tumor cells in exosomes. Cancer Res 2010;70:481-9.

[94] Valenti R, Huber V, Filipazzi P, Pilla L, Sovena G, Villa A, et al. Human tumorreleased microvesicles promote the differentiation of myeloid cells with transforming growth factor-beta-mediated suppressive activity on $\mathrm{T}$ lymphocytes. Cancer Res 2006;66:9290-8.

[95] Xiang X, Poliakov A, Liu C, Liu Y, Deng ZB, Wang J, et al. Induction of myeloidderived suppressor cells by tumor exosomes. Int J Cancer 2009;124:2621-33.

[96] Hood JL, Pan H, Lanza GM, Wickline SA. Paracrine induction of endothelium by tumor exosomes. Lab Invest 2009;89:1317-28.

[97] Park JE, Tan HS, Datta A, Lai RC, Zhang H, Meng W, et al. Hypoxic tumor cell modulates its microenvironment to enhance angiogenic and metastatic potential by secretion of proteins and exosomes. Mol Cell Proteomics 2010; 9:1085-99.

[98] Hong BS, Cho JH, Kim H, Choi EJ, Rho S, Kim J, et al. Colorectal cancer cellderived microvesicles are enriched in cell cycle-related mRNAs that promote proliferation of endothelial cells. BMC Genomics 2009;10:556.

[99] Bhatnagar S, Schorey JS. Exosomes released from infected macrophages contain Mycobacterium avium glycopeptidolipids and are proinflammatory. J Biol Chem 2007;282:25779-89.

[100] Sun D, Zhuang X, Xiang X, Liu Y, Zhang S, Liu C, et al. A novel nanoparticle drug delivery system: the anti-inflammatory activity of curcumin is enhanced when encapsulated in exosomes. Mol Ther 18:1606-14.

[101] Laulagnier K, Grand D, Dujardin A, Hamdi S, Vincent-Schneider H, Lankar D, et al. PLD2 is enriched on exosomes and its activity is correlated to the release of exosomes. FEBS Lett 2004;572:11-4.

[102] Chalmin F, Ladoire S, Mignot G, Vincent J, Bruchard M, Remy-Martin JP, et al. Membrane-associated Hsp72 from tumor-derived exosomes mediates STAT3dependent immunosuppressive function of mouse and human myeloid-derived suppressor cells. J Clin Invest 2010;120:457-71.

[103] Sertznig P, Seifert M, Tilgen W, Reichrath J. Present concepts and future outlook: function of peroxisome proliferator-activated receptors (PPARs) for pathogenesis, progression, and therapy of cancer. J Cell Physiol 2007;212:1-12.

[104] Scott SA, Selvy PE, Buck JR, Cho HP, Criswell TL, Thomas AL, et al. Design of isoform-selective phospholipase D inhibitors that modulate cancer cell invasiveness. Nat Chem Biol 2009;5:108-17. 
[105] Xie Y, Bai O, Zhang H, Yuan J, Zong S, Chibbar R, et al. Membrane-bound HSP70engineered myeloma cell-derived exosomes stimulate more efficient CD8(+) CTLand NK-mediated antitumour immunity than exosomes released from heat-shocked tumour cells expressing cytoplasmic HSP70. J Cell Mol Med 2010; 14:2655-66.

[106] de Medina P, Paillasse MR, Payre B, Silvente-Poirot S, Poirot M. Synthesis of new alkylaminooxysterols with potent cell differentiating activities: identification of leads for the treatment of cancer and neurodegenerative diseases. $J$ Med Chem 2009;52:7765-77.

[107] Silvente-Poirot S, Record M, de Medina P, Subra C, Payré B, Paillasse M.R, Poirot M. . Dendrogenin A modifies agressive melanoma cells to secrete exosomes with antitumor activity. Abstract. International Worshop on Exosomes, 2011, January 19-22, Paris, France 2011.

[108] Thery C, Amigorena S, Raposo G, Clayton A. Isolation and characterization of exosomes from cell culture supernatants and biological fluids. Curr Protoc Cell Biol 2006; Chapter 3:Unit 322.

\section{Figure Legends}

Figure 1. The "exosome pathway". Intralumenal vesicles (ILV) are formed in viable cells in a subset of late endosomes called MultiVesicular Bodies (MVB) (A). Once released outside the cells these vesicles are called "exosomes" (B) which can be taken up by target cells (C) or be swept away by the blood stream or by other biological fluids (D).

Letters A-D also refer to the chapters of this review.

Figure 2. Example of lipid sorting in MVB and exosomes. The lysophospholipid named LBPA (lysobis-phosphatidic acid), also called BMP (bis-(monoacylglycero)-phosphate) starts to appear at the stage of MVB along the endocytic track, whereas its precursor, phosphatidylglycerol (PG) is biosynthesized in the mitochondria. Exogenous PG can be converted into LBPA in the MVB [28]. LBPA regulates the ILV turn-over within the MVB [27]. Bioactive lipids such as prostaglandins are enriched in exosomes and are thus transported outside the cells [9]. The 3 families of phospholipases A2 and the COXs (cyclo-oxygenase 1 and 2) are embedded in exosomes [9]

Figure 3. Constitutive secretion of exosomes from $\mathrm{T}$ cell hybridomas. Enhanced constitutive exosome production was observed when $T$ cell hybridomas (2B4) were transduced by the retroviral particles containing LXSP-PLD2 construct. LXSP was derived from Murine Moloney Leukemia retroVirus (MMLV). LXSP-PLD2 construct contained the human phospholipase D2 (PLD2) sequence and was packaged as a transcript into infectious particles derived from BOSC packaging cells. As shown for the regulated pathway (Fig 1 and reference[101]), PLD2 enhanced secretion of exosomes. Carrier vesicles containing only one or two exosome were formed from the tubulovesicular system (arrows 1,2) and next fused with the plasma membrane, allowing the release of exosomes (arrow 3), which accumulated between proximal cells (arrow 4). Some of the vesicles contained material with high density to electron transmission (inset in [4]) which evoked a viral capsid formation, which would 
originate from the retroviral transduction of cells. This suggests that both exosomes and retroviral particles might be produced at the same time (M.R. unpublished observations).

\section{Figure 4. Some proteins and lipids present in exosomes.}

About a hundred fold enrichment of tetraspanins (CD9, CD63, CD81, CD82) in exosomes comparatively to parent cells is a hallmark of exosomes [10]. Heat shock proteins (Hsp 90, Hsp 70, Hsc 70) also are enriched. Activation of phospholipases by GTPases (Rab, Rap, ran, RhoA, Arf) and their regulation by aldolase, casein kinase II, and Hsp/Hsc 70 has been reported in the literature and in reference [9]. The major phospholipids are present in exosomes but in distinct proportions as compared to parent cells, and lipid rafts have been characterized. The specific MVB lyso-lipid LBPA (BMP) is also found in exosomes . 
Figure 1 M.Record

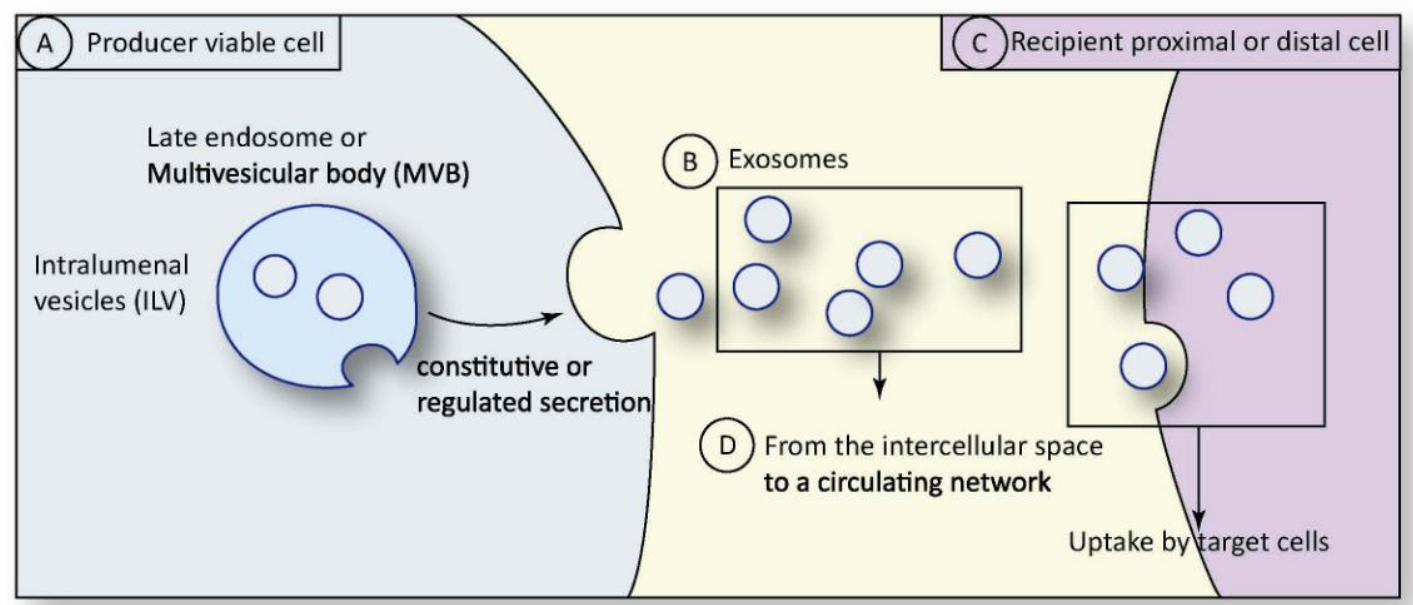




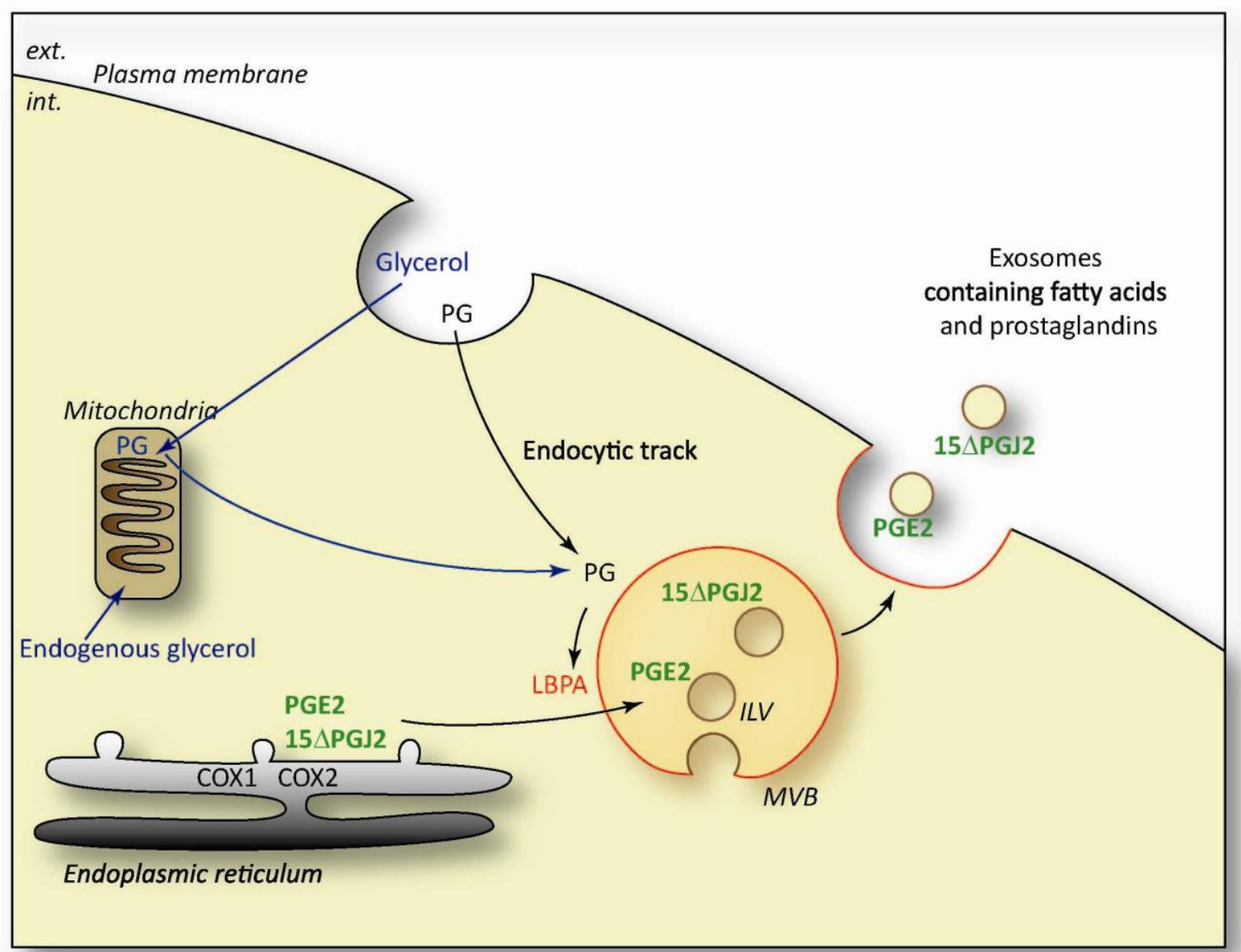




\section{Figure 3 M.Record}

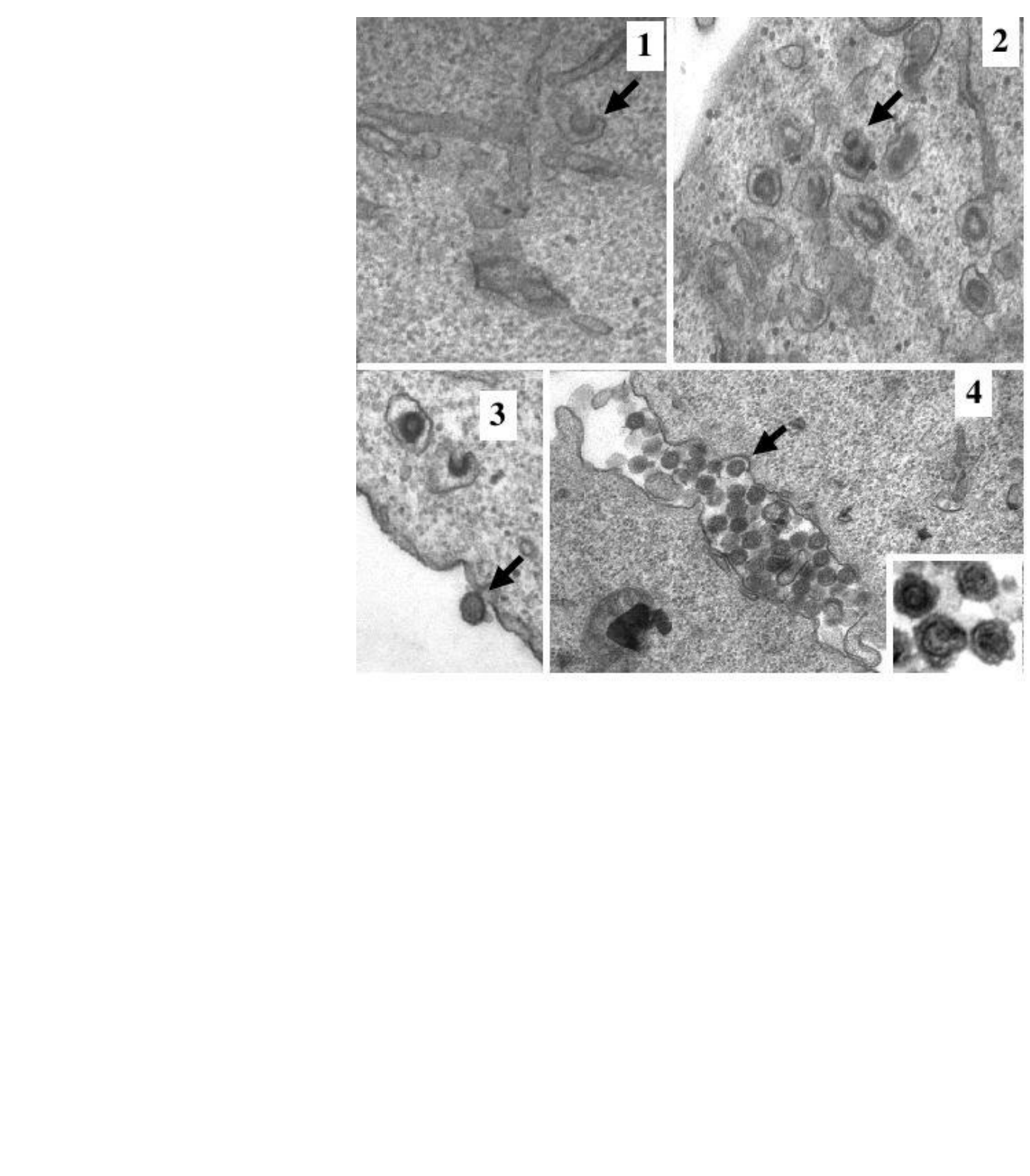

Figure 
Figure 4 M.Record

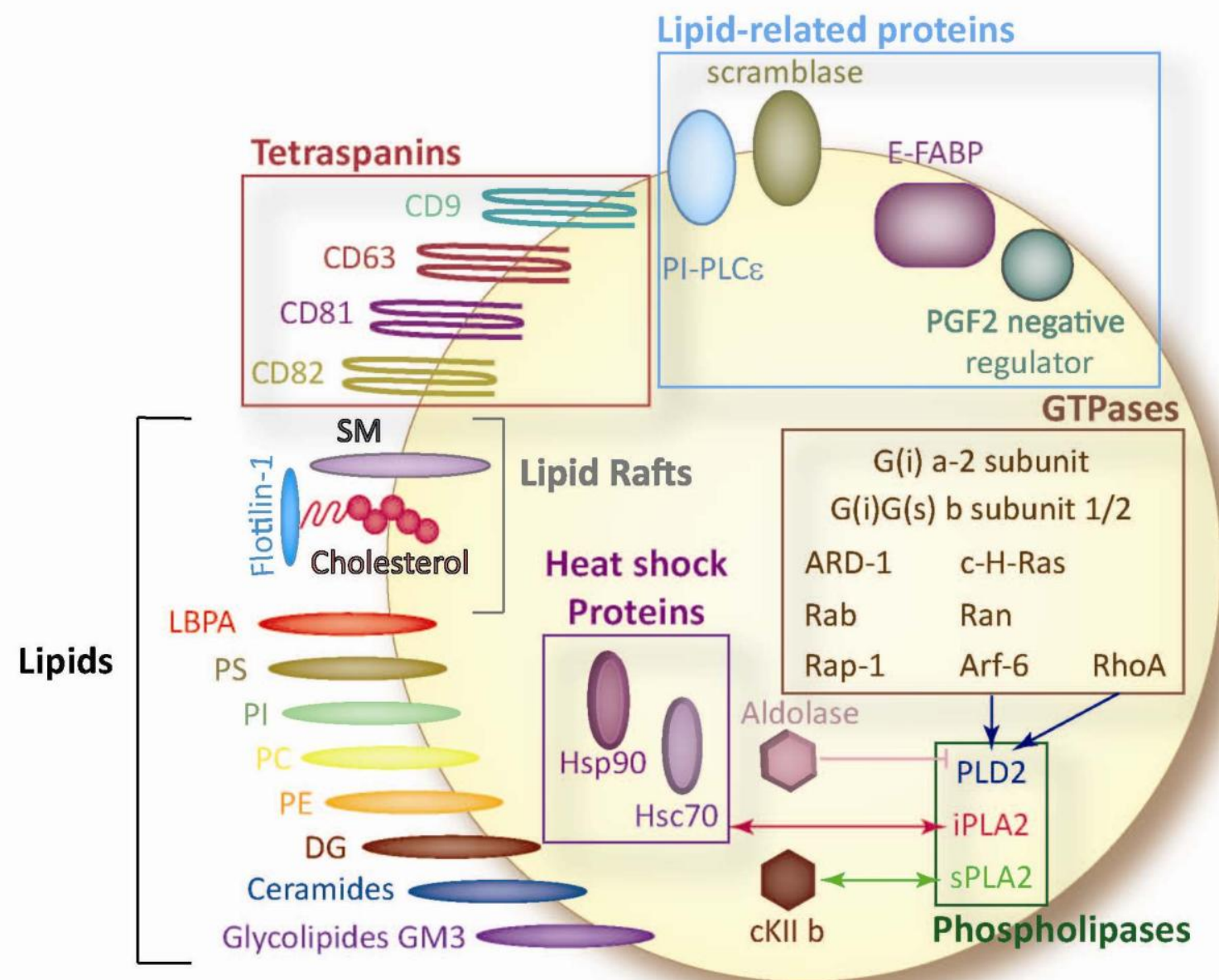




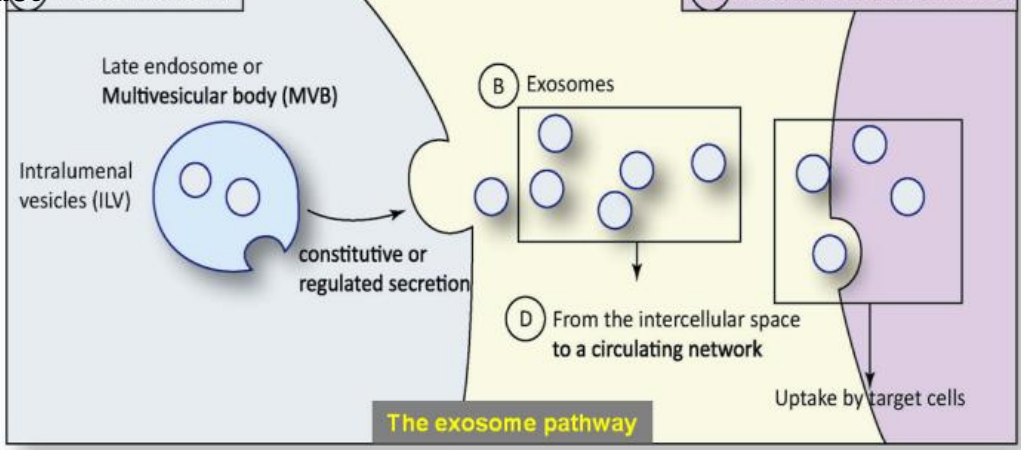

